\title{
Using Intelligent Agents for Building Evacuation: A Uml Approach
}

\author{
Gurpreet Singh \\ Department of C.S.E \\ Sri Sai college of Engg \\ Badhani, Pathankot
}

\author{
Prof. Menakshi Sharma \\ Department of C.S.E \\ Sri Sai College of Engg \\ Badhani,Pathankot
}

\author{
Prof. Amitpal Singh \\ Department of C.S.E \\ G.N.D.U Regional campus \\ Gurdaspur
}

\begin{abstract}
Over the last decade applications of agent based system has increased rapidly because agents have number of abilities such as act, sense and reason according to situation. Intelligent agent perceives its environment and takes actions to maximize its chances of success. In this paper UML (unified modeling language) has been used to design a building evacuation system in which agents has been used as experiment in real cannot be performed. It also shows how UML can be exploited to design multi agent system at agent level. It provides different agent oriented diagrams to model architecture of multi agent system and coordination between agents. Agents have been modeled in different situations and the way they think and how they use the available resources for their exit.
\end{abstract}

Keywords

Agents, UML, building evacuation, sensors, detectors

\section{INTRODUCTION}

Artificial intelligence is the science and engineering of making intelligent machines which is concerned in making computers to behave like humans .Artificial intelligence includes

- Games playing: programming computers to play games

- Experts systems: : programming computers to make decisions in real-life situations

- Natural language : programming computers to understand natural human languages

- Neural networks : Systems that simulate intelligence by attempting to reproduce the types of physical connections that occur in animal brains

- $\quad$ Robotics : programming computers which helps see and hear

It is the study and designing of intelligent machines [1]. An intelligent machine is a system that perceives its environment and takes action to maximize its chances of success. The concept of agents first appeared in seventies due to an increasingly large number of applications, we require a system that can decide for themselves such computer system are known as agents today we find vast applications of agents in different fields of life in order to reduce workload on human beings and make the process more efficient[2][3]. Agents can act and think in more efficient way as compare to human beings in difficult and extreme situations where human's cannot work efficiently. Some agents have limited knowledge i.e they can only do work for which they have been made they do not learn new things according to situation and environment and some learn from the environment and have the ability to think and learn new things according to situation and environment. Intelligent agents have mind which are capable of choice they can look at situation and choose from a number of possible courses of action to pick one which solves the problem [3][4]. In this paper concept of multi agent system has been used which consist of number of agents which interact with one another it is a loosely coupled network of problem solvers that interact with each other to solve a problem that are beyond the individual capabilities When interdependent problem arise the agents coordinate with each other to solve that particular problem. Every agent has incomplete knowledge and information about the environment and situation their emphasis is to decompose the problem and then solve it and in order to interact they required to cooperate, coordinate and negotiate with each other[5][6][12]. Extensions proposed in this paper are used for modeling agent based system .Agent class diagrams which describes the static structure of the system by showing the system classes their attributes, operations and relationship among classes. Agent collaboration diagrams which describes dynamic behavior of the system and interaction among objects. They are the another form of interaction diagram they represents objects and links. They also describe the message flow in the system [7][8]. The aim of the paper is to develop a building evacuation system using agent based modeling technique and UML no matter what type of building it is(a residential society, a school, hospital or bank) is not a parameter which is to be considered while the evacuation of building.

\section{PROPOSED APPROACH}

In previous work [16] evaluation on building security has been performed by using agents on single room and multiple rooms only and how agents evacuate from building by their own. We are extending this model for building evacuation in case of any disaster occurrence, by showing all the available exits, which agents can use for their exit and different agents have been used in this approach which collectively act as rescue team (multi agent system), in which each agent has been assigned a particular responsibility which it has to serve. Also, how various agents communicate with each other to perform building evacuation process is shown and has been represented by using collaboration diagrams. In this proposed work we suggest an agent based modeling technique for the simulation of a building evacuation (in case the building is on fire).While designing the building this modeling can help in various architectural decisions and to show interaction among various emergency services which may affect evacuation of building when some emergency occurs, such as placement of regular and emergency door, situation of stair case and lift in building and room to room connectivity. In this case, this type of simulation (through modeling) is only choice as direct experiment (real evacuation) cannot be performed. We propose the use of AUML (extended Agent based UML) for modeling Agent Diagrams(similar to object diagrams), Agent Class Diagrams(similar to class diagrams), Agent Collaboration Diagrams(similar to collaboration diagrams) for 
expressing agent capabilities and agent interactions in order to perform the job of building evacuation using software agents[12][15][16].

\section{EXTENDING UML APPROACH FOR MODELING AGENTS}

UML stands for Unified Modeling Language. UML is a pictorial language used to make software blue prints. In 1997 OMG (object management group) develop UML as a common architectural framework for modeling systems and applications. The UML uses mostly graphical notations to express the Object Oriented analysis and design of software projects. It helps to acquire an overall view of a system. UML is not dependent on any other language or technology it does provide several types of diagrams that, when used within a given methodology, increase the ease of understanding an application [7][10].
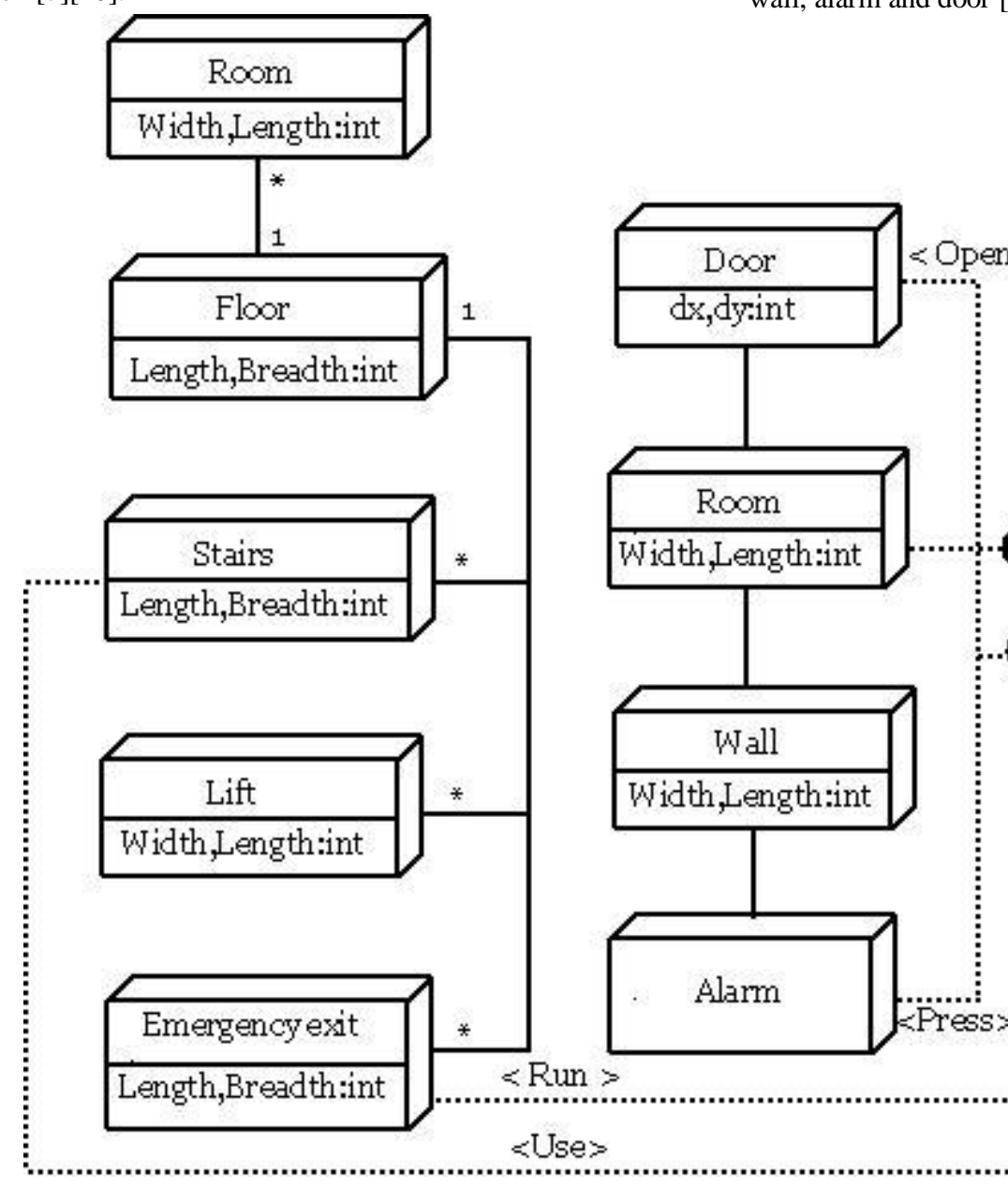

Figure 1: Class diagram of moving agent

The class diagram in Figure 2 shows evacuation procedure during emergency situation or when the building is on fire. Any agent who identifies the emergency situation press emergency alarm button which are located throughout the building. There are two alarm signals which sound through the whole building one is alert tone the other one is evacuation tone. When Alert tone is pressed this tone is an indication of emergency situation has occurred and be ready to evacuate they are required to wait at their places for further

\subsection{Agent Class Diagrams}

A class diagram in the Unified Modeling Language (UML) represents static structure of the system system's classes, their attributes, operations (or methods), and the relationships among the classes The class diagram in Figure 1 is a type of static structure diagram that describes the structure of a system by showing the system's classes here is a class diagram of moving agent of an agent class runner which has a Sensor (View) and Effector (React). The agents can sense doors, walls and other agents through its sensors and have the ability such as they can open or close doors, press alarm and act according to the situation with the help of effectors. They can use various options for exit such as stairs, lift and emergency exit. Agents capabilities are specified in agent class box sense and act. In above diagram different exit options are shown on a single floor such as it is having lift, stairs, emergency exit and room. While attributes of room is shown which is having wall, alarm and door [4][11]. 


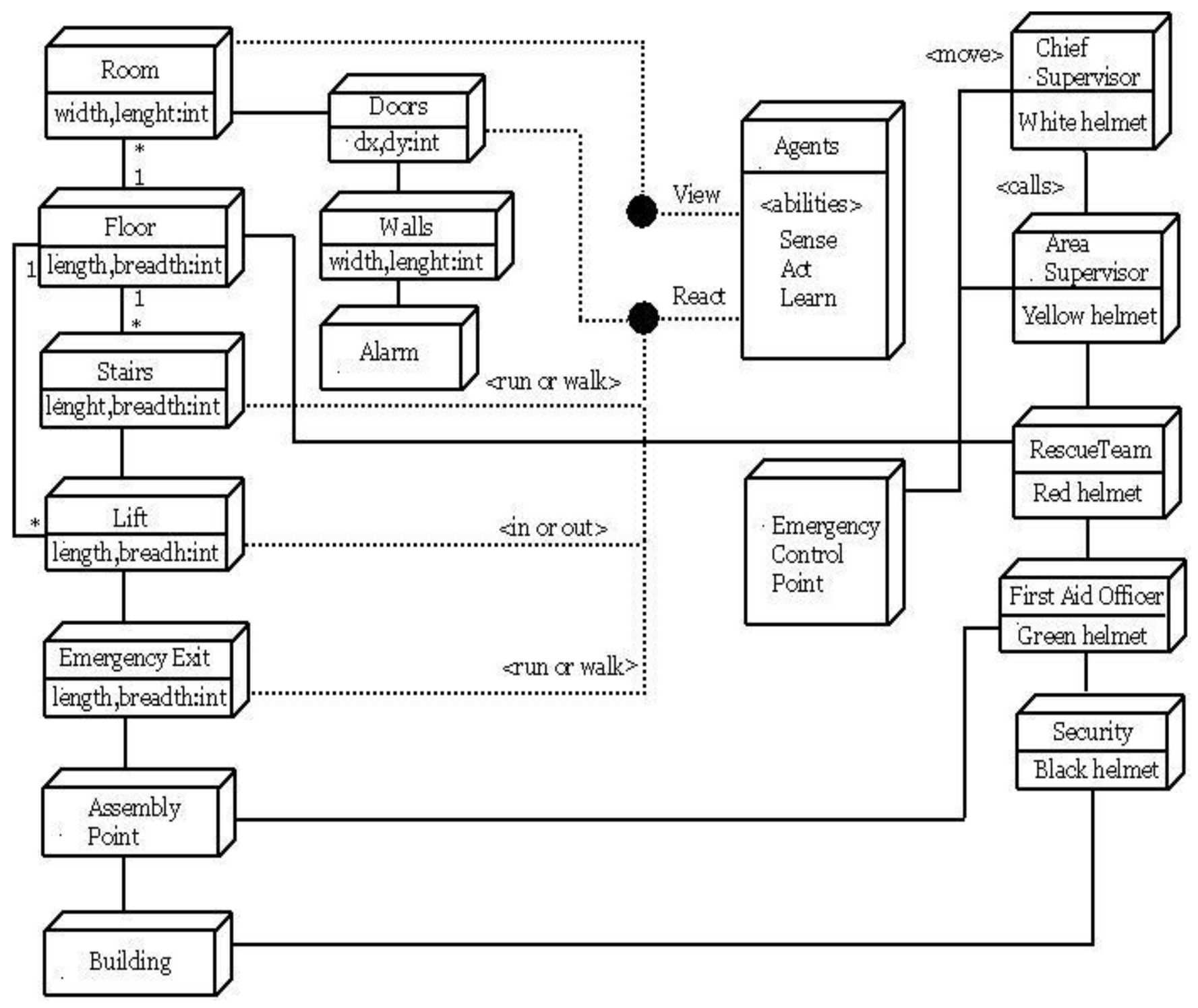

Figure 2: Evacuation Class Diagram

two way radio. Area supervisor is supposed to proceed to effected area and contact chief supervisor and make him aware about the situation and wait for further instruction from chief supervisor. Rescue team will gather their equipments and move to effected area they help to find exit routes from the building and evacuating injured persons. First aid officer will move to assembly point and provide assistance to people's until medical team arrives. Security personnel have to ensure that all fires exits are not locked and ensures that no one re enters the building except rescue team. Structure of building consist of a room which is having doors, walls and alarm on the other side a floor is there which is having stairs, rooms, lifts and emergency exit the spatial dimensions of all these are stored in attributes width and length. After evacuation proceed to assembly point so that it is convenient to notify if any body is missing or left back in the building it can by reviewing the record of the visitors who are staying in building if somebody is still missing they can again search him to rescue[4][11][15].

\subsection{Agent Collaboration Diagrams}

A collaboration diagram describes interactions among objects in terms of sequenced messages. They are also known as interaction diagram they show how object interact over the In Figure: 3 the collaboration diagram specify the role of Chief supervisor who is in white helmet immediately after the activation of emergency evacuation alarm he will assume the control of situation until he handover control to emergency service personnel. He will then move to emergency control point in order to prepare evacuation plan. Then concerned area warden will be contacted from where alarm has been activated and other authorities should be notified such as Police, fire brigade and ambulance and to assist them as per need. They have ensure that everyone has been evacuated from the building and advise everyone when it is safe to reenter the building. 


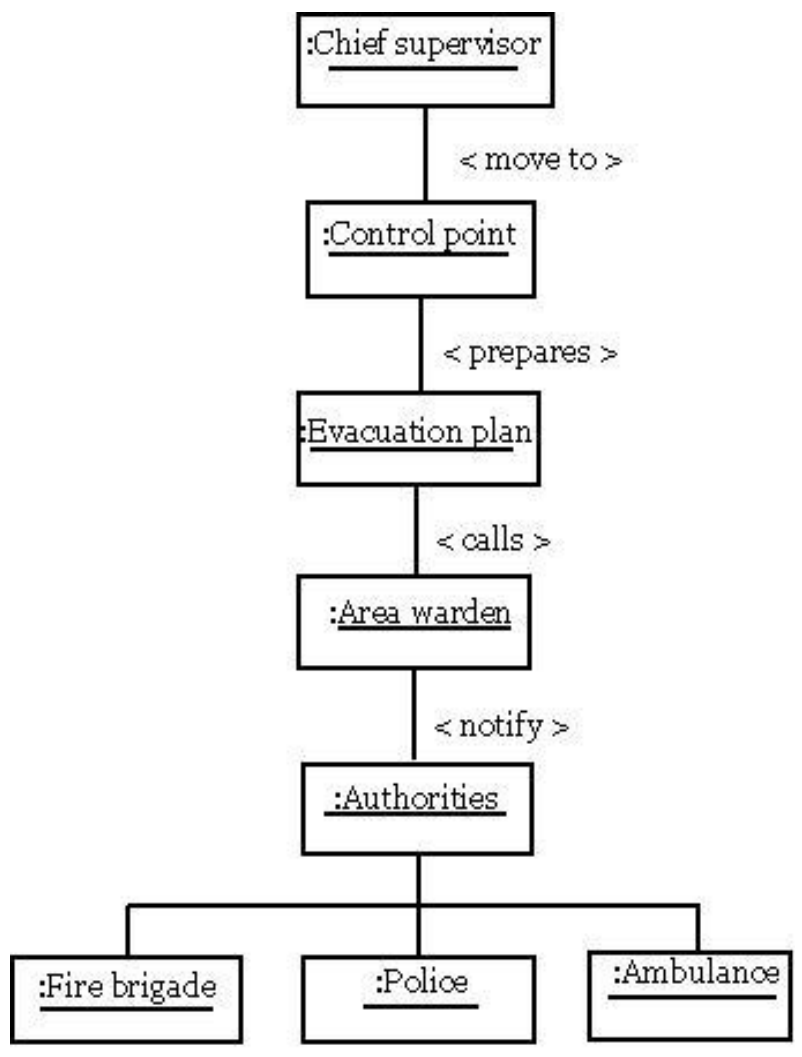

Figure3: Collaboration diagram specifying responsibilities and functions of Chief Supervisor

In Figure 4 Area supervisor in the event of emergency takes control of his allocated area upon hearing the alarm will contact Chief supervisor who will instruct him by two way radio. In case the chief supervisor is absent area supervisor have to assume the whole control of the situation and must be familiar of the responsibilities of chief supervisor .If needed he is supposed to provide necessary equipments such as first aid box or tools to rescue teams. It will then move to his zone will then check their designated zone and report to chief warden about the situation and when ordered to evacuate by him or when evacuation signal sounds facilitate the evacuation of the area. Search the zone to make sure that everyone has left the area and personally check the rooms, office and washrooms to ensure that everyone has left the area and collect the visitor register sheet from reception desk and check whether all the persons in building are present or not in the event of missing person notify the rescue team who will again search building. When zone is cleared report back to chief supervisor.

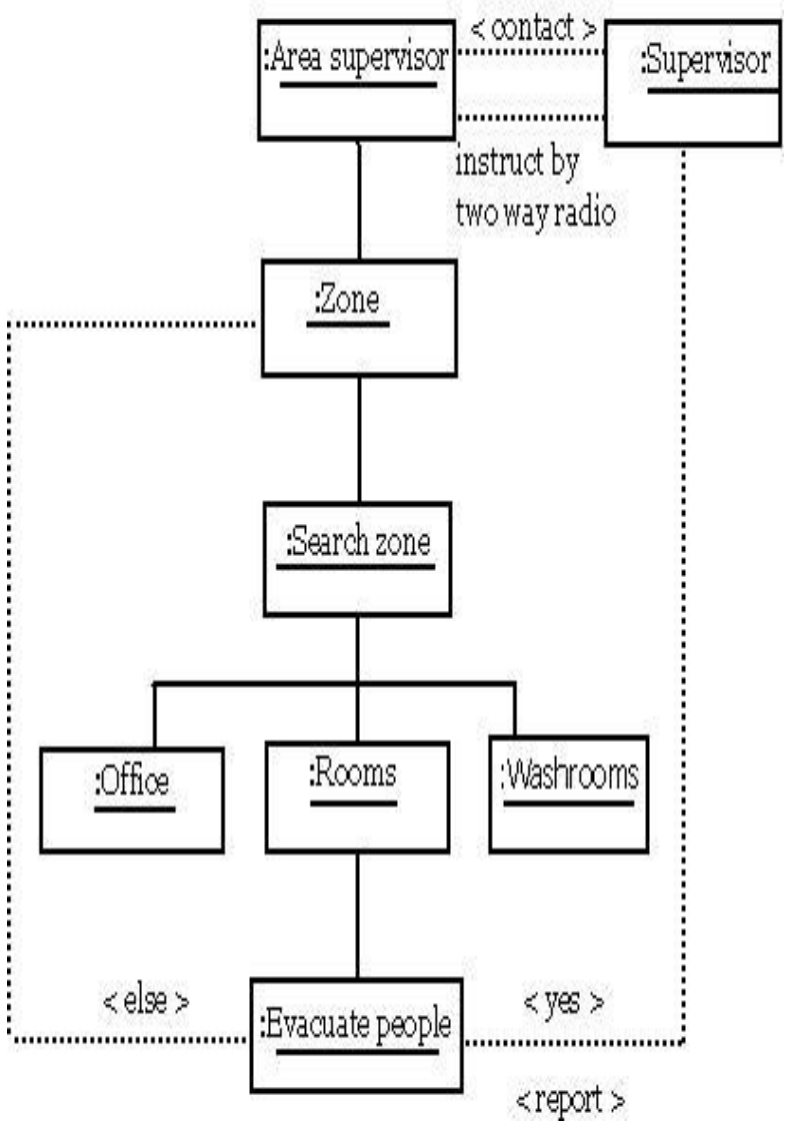

Figure4: Collaboration diagram specifying responsibilities and functions of Area supervisor

In Figure 5 Rescue team will be in red hat in the event of emergency they help area wardens in rescue operation upon hearing alert tone gather their equipments collect all the necessary information from the area wardens and move to effected area. They have to assist people in the evacuation through their nearest emergency exit and assist in the evacuation of people with disabilities. In case fire exits are locked or blocked they have to unlock or clear exits as soon as possible. Instruct first aid officer to provide assistance to injured people until medical team or ambulance arrives there upon moving those injured persons to hospital report to area warden then again check the whole building so that no body is left over there and mark that area clear zone they instruct everyone when it is safe to re enter the building[10][15]. 


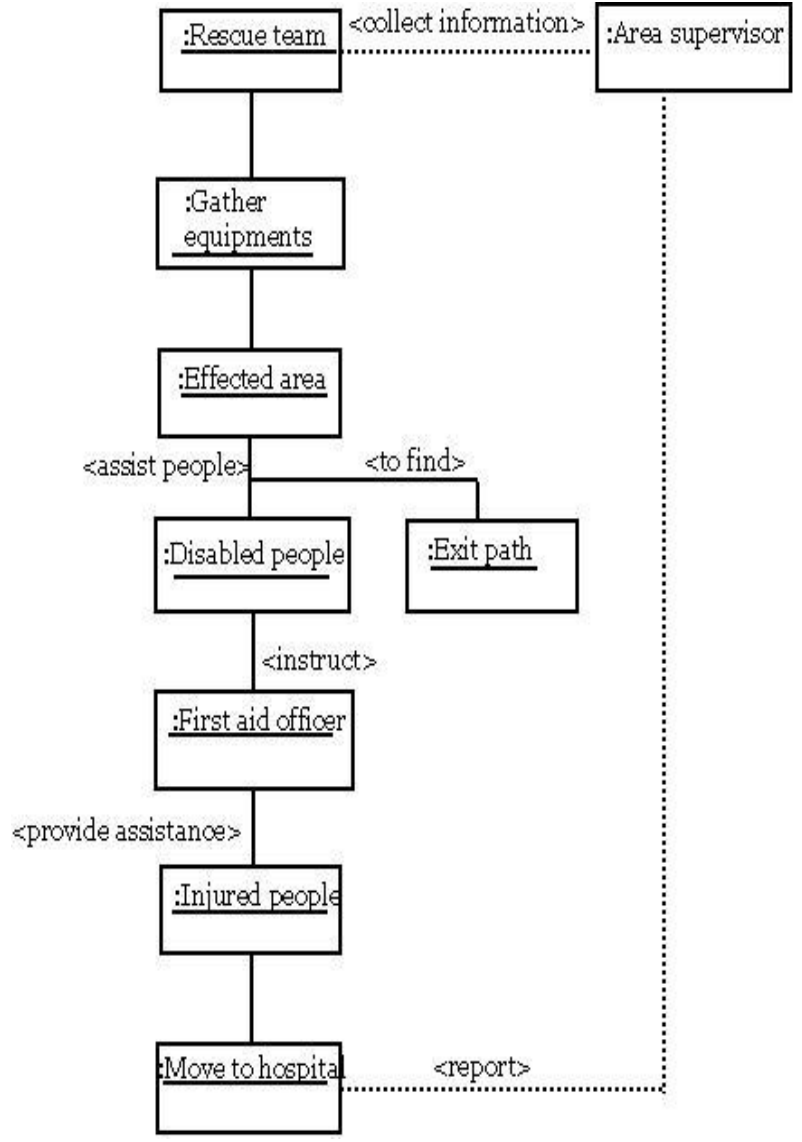

Figure5: Collaboration diagram specifying responsibilities and functions of Rescue Team

\section{EVACUATION PROCEDURE}

Any person who identify an emergency situation must do the following things depending upon the situation:

1.Press emergency alarm button located throughout the building

2. Dial reception

3. Contact security, chief supervisor or area supervisor.

4. Dial emergency services

In this model two alarm tones have been used one is alert and second one is for evacuation. Alert tone is used for indicating the emergency and prepare or get ready to evacuate during this time they are required to wait for further instruction they are not supposed to leave their places. The second alarm tone is used for evacuation which indicates building evacuation is required this indicate everyone is supposed to leave the building. In case alarm is false chief supervisor announce it's a false alarm and every body should return back to their place. Then walk to the nearest safe external exit do not run. People should not close the main doors behind from where they are passing only close their room and make sure that no body is left behind. They should not stop and pick their luggage or articles. During evacuation one must follow or do these things

- One should know all fires exit or routes from the building.

- Dial emergency during any smoke or smell.

- Only use fire exit for evacuation.

- Do not run or use lifts.

- Leave fire escape doors open.

- Gather to assembly point located at the base of the building

- Report about any missing person to concerned authority

\section{CONCLUSION AND FUTURE WORK}

In this paper we proposed a model of building evacuation system and also provided a possible solution to the problem of building evacuation during emergencies and working of various emergency services along with their roles with the help of UML using intelligent agents. As in this life -critical case, real experiment cannot be performed by humans, So by simulating this model using any MAS simulator, we can further make our evacuation system better and moreover we can enhance our building securities also with this model. This model can be developed further to be implemented in real .. This model can be further modified or extended to work with various different types of evacuation plans (on-site and offsite) for other different types of disasters (natural and artificial), like earthquakes, cyclones, floods, landslides etc for building evacuation and shifting to a safe place or shelter in the case of their occurrence

\section{REFERENCES}

[1] Stuart Russell and Peter Norvig. Artificial Intelligence Prentice-Hall, Inc. 1995

[2] James Odell. Introduction to agents 2000.

[3] Wooldridge M.J., Jennings N., Intelligent Agents: Theory and Practice, Knowledge Engineering Review, 1995, 10, 115-152.

[4] N.R .Jennings and M. Wooldridge Applications of Intelligent Agents, 1995.

[5] Brad Clement. Multiagent planning, Artificial Intelligence Group Jet Propulsion Laboratory California Institute of Technology" http://ai.jpl.nasa.gov/.

[6] Gerhard Weiss. Multi agent Systems. Massachusetts Institute of Technology London, England The MIT Press Cambridge, Massachusetts1999.

[7] www.tutorialspoint.com/index.html.

[8] Dan brown, Dunstan Thomas. A beginners guide to UML Part 1.

[9] Dan brown, Dunstan Thomas .A beginners guide to UML part 2.

[10] www.aUML.org

[11] F. Bergenti, A. Poggi. Exploiting UML in the design of Multi-Agent systems Proceeding of the ECOOP Workshop on Engineering Societies in the Agents World 2000 (ESAW 00), pp. 96-103, 2000

[12] Katia Sycara. MultiAgent Systems. AI Magazine 19(2): Summer 1998, 79-92

[13] Eduardo Alonso. AI and Agents State of the art .AI Magazine 23(3): Fall 2002,

[14] Eliezer Yudkowsky Artificial Intelligence as a Positive and Negative Factor in Global Risk Singularity Institute for Artificial Intelligence Palo Alto, CA. Draft of August 31,2006

[15] Krishna Kavi, David C Kung, Hitesh Bhambhani, Extending UML for modeling and design of multi agent system.

[16] Juan De Lara Modeling agents with UML : an example to building security evaluation. 